\title{
Review of: "Better COVID-19 Intensive Care Unit survival in females, independent of age, disease severity, comorbidities, and treatment"
}

ekaterini goudouris

Potential competing interests: The author(s) declared that no potential competing interests exist.

Great paper, well done piece of work and nicely written. It brings important information about sex susceptibility that needs to be further explored in order to disclosure new treatment possibilities or even selective prevention measures. 